\title{
Multidimensionally resolved pore size distributions by NMR
}

\author{
J H Strange and J B W Webber
}

Physics Laboratory, University of Kent, Canterbury CT2 7NR, United Kingdom.

\begin{abstract}
.
We have developed a novel method of determining median pore size and pore size distributions as a function of spatial position inside a porous sample.

Pore sizes have been measured with 1,2 and 3-dimensional spatial resolution, using NMR Cryoporometry in conjunction with magnetic resonance imaging techniques.

NMR Cryoporometry is a method of measuring pore sizes and pore size distributions in the range of less than $40 \AA$ to over $2000 \AA$ pore diameter, by the technique of freezing a liquid in the pores and measuring the melting temperature by Nuclear Magnetic Resonance. Since the melting point is depressed for crystals of small size, the melting point depression gives a measurement of pore size.
\end{abstract}

(for Measurement Science and Technology)

2500 words 


\section{NMR Cryoporometry}

Porous materials occur widely and have many important applications. They can, for example, offer a convenient method of imposing fine structure on adsorbed materials. They can be used as substrates to support catalysts and can act as highly selective sieves or cages that only allow access to molecules up to a certain size. Many biologically active materials are porous.

Commercially important porous materials include the porous glasses, the alumina and alumino silicates such as clays and zeolites, activated and other porous carbons, and water and oil bearing shales, sandstones and limestones, as well as many construction and engineering materials. Porous materials play an important part in many biological processes and life forms.

Characterisation and measurement of porous media is therefore of considerable interest, and NMR cryoporometry may be a useful method to add to existing porous measurment techniques such as gas adsorption and mercury porosimetry. It shows good linear agreement with gas adsorption measurements in porous silica glasses, up to the currently demonstrated maximum pore diameter of $2000 \AA$. Where NMR certainly has an advantage is in its ability to non-destructively spatially resolve pore size information, as shown here.

We may define porosity as the fraction of the sample volume occupied by pore void; an important characterising parameter is the porosity as a function of pore diameter and spatial location, i.e. the poresize distribution within a material.

Recent work by Jackson and McKenna [1] demonstrated that the melting point depression of a variety of liquids confined in silica glasses varied inversely with the mean pore diameter as determined by gas adsorption isotherm measurements.

This behaviour is closely related to the capillary effect; both reflect the change in free energy caused by the curvature of the liquid surface.

The Gibbs-Thomson equation for the melting point depression $\Delta \mathrm{T}_{m}$ for a small crystal of diameter $\mathrm{D}$ is given $[1,2]$ by :

$$
\begin{aligned}
\Delta \mathrm{T}_{m}= & \mathrm{T}_{m}-\mathrm{T}_{m}(\mathrm{D})=4 \sigma_{s l} \mathrm{~T}_{m} /\left(\mathrm{D} \Delta \mathrm{H}_{f} \rho_{s}\right) \\
\text { where : } & =\text { normal melting point of bulk liquid } \\
\mathrm{T}_{m} & =\text { melting point of crystals of diameter } \mathrm{D} \\
\mathrm{T}_{m}(\mathrm{D}) & =\text { surface energy at the liquid-solid interface } \\
\sigma_{s l} & =\text { bulk enthalpy of fusion } \\
\Delta \mathrm{H}_{f} & =\text { density of the solid }
\end{aligned}
$$

To exploit this effect for pore size measurement a porous sample containing a liquid is cooled until all the liquid is frozen, and then gradually warmed while monitoring the 
amplitude of the NMR proton spin echo from any liquid present. The liquid is usually chosen to be water or cyclohexane, the latter offering the large melting point depression of $1825 \mathrm{~K} \AA$. i.e. a depression of nearly $20 \mathrm{~K}$ in $100 \AA$ pores.

NMR is a sensitive technique for distinguishing between solid and liquid, as the coherent transverse nuclear spin magnetisation decays much more rapidly in a solid than in a mobile liquid. Its measurement is usually most conveniently done using a $90^{\circ}{ }^{-}-\tau-180^{\circ}{ }_{y}$ - $\tau$-echo sequence $[2,3]$, where the time interval $2 \tau$ is set to be longer than the solid decay time but less than the decay time in the liquid, and is typically around $20 \mathrm{~ms}$. Thus the amplitude of the echo records the amount of solid that has melted to a liquid at a particular temperature.

Since this echo amplitude should only increase as the sample warms up, the constraint of monoticity is applied to the data. To obtain a pore size distribution, i.e. porosity measured as a funcion of poresize, the echo amplitude is differentiated as a function of temperature :

$$
\text { SlopeOfEchoAmplitude }=\frac{d(\text { EchoAmplitude })}{d(\text { Temperature })}
$$

This is then remapped as given by the Gibbs-Thomson equation : a new $\mathrm{X}$ value is calculated from :

$$
\frac{\text { CalibrationSlope }}{\text { BulkMeltingPoint-Temperature }}
$$

while the $\mathrm{Y}$ value is rescaled :

$$
\text { (BulkMeltingPoint-Temperature })^{2} \times \text { SlopeOfEchoAmplitude }
$$

This has two effects: the new $\mathrm{X}$ ordinates are wildly non umiform in spacing, being extremely dense at small pore sizes, and widely spaced at large pore sixes; and the noise is amplified hugely at small pore sizes. Clearly the change in point density can be used to counter the non uniformity in noise, and, making use of this behaviour, a logarithmically spaced smoothing is applied to the transformed data.

We have been developing this technique in our laboratories [2,4], and have recently combined it with magnetic resonance imaging techniques to obtain spatially resolved pore size distributions. Thus we demonstrate here the use of nuclear magnetic resonance as a convenient technique for measuring the fraction that is liquid, throughout the porous material, and as a way of spatially encoding the porosity, to produce a pore size distribution map. 


\section{Experimental arrangement}

\section{1. $1 D$ Measurements}

The solid state NMR relaxation spectrometer used was designed and constructed by us. The magnet was a $21.5 \mathrm{MHz}$ (protons) Mullard ${ }^{T M}$ permanent magnet, with a $35 \mathrm{~mm}$ gap, into which was inserted a Dewar containing the NMR probe, with cooling by direct injection of liquid nitrogen [5].

\subsection{D and 3D Measurements}

For the two-dimensional and three-dimensional measurements, the spectrometer used was a modified Bruker ${ }^{T M}$ CXP200 console interfaced by us to an industrial grade 486 DX33 personal computer running SMIS ${ }^{T M}$ software and with SMIS plug in modules. The magnet used was a $200 \mathrm{~mm}$ horizontal bore Magnex ${ }^{T M}$ superconducting magnet, operating at $105 \mathrm{MHz}$ for protons.

\section{1D NMR Cryoporometry}

Test samples were prepared (Figure 1a), in $5 \mathrm{~mm}$ diameter tubes, each consisting of three layers of porous silica of $60 \AA, 140 \AA$ and $500 \AA$ nominal pore diameter, separated by PolyTetraFluroEthane spacers (giving no proton signal). Water or cyclohexane was added to the silica, to give approximately $110 \%$ filling of the pores. i.e. $10 \%$ more liquid was added to the porius media than necessary to just fully fill the pore volume, the excess going to fill the

inter-particle space.

To obtain spatial resolution of the pore size distribution phase and/or frequency encoding of the NMR signal [6] may be used. In the case of 1D Cryoporometry [4], the main $\mathrm{B}_{0}$ field was applied along the $\mathrm{z}$ axis, and a constant magnetic field gradient $\mathrm{G}_{y}=$ $\delta \mathrm{B}_{z} / \delta \mathrm{y}$ was applied in the perpendicular y direction along the axis of the NMR sample tube, to obtain frequency encoding of the axial position. The gradient was generated by four current carrying wires plus return wires, suitably spaced [7] to minimise all but the linear gradient term $\mathrm{G}_{y}$. Since the NMR precession frequency is proportional to the magnetic field $B_{0}$, the frequency of the NMR signal varies linearly with axial position.

Rather than recording just the peak amplitude of the echo at each temperature as in normal NMR cryoporometry, the complete echo envelope was captured. Since the linear field-gradient allows a linear mapping from frequency space to y-dimension, the Fourier transform of this echo then gives a $1 \mathrm{D}$ profile of the liquid component at this temperature.

To make a measurement the test sample was cooled until all the liquid was frozen, 
and there was no signal. It was then slowly allowed to warm naturally in the gradient. A succession of 1D profiles was captured, averaging 16 times, as the sample warmed. The liquid in the $60 \AA$ silica melted first, then that in the $140 \AA$ silica, followed by that in the $500 \AA$ silica, and finally any bulk liquid outside the pores. When the temperature run was complete, the standard cryoporometric differentiation and re-mapping was performed along the temperature axis, for each array point in the sample.

This re-mapped data is presented as a surface map, and as an intensity map (figure 1b), giving the porosity within the sample as a function of pore diameter and axial position. This shows that the porosity is successfully resolved with good spatial resolution and pore size resolution comparable with that from a standard cryoporometry run. The median pore size was also calculated, for each point in the $1 \mathrm{D}$ sample (figure $1 b$, lower right).

\section{2D Resolved Pore Size Distributions}

A test phantom (figure 2a) was constructed, consisting of four $5 \mathrm{~mm}$ high-resolution thin walled glass tubes, containing $40 \AA, 60 \AA, 140 \AA$, and $200 \AA$ pore diameter porous silica, arranged in a square array.

The sample was cooled with liquid nitrogen, and 32 images were recorded as a function of temperature, as the phantom warmed up naturally. At each temperature, a $2 \mathrm{D}$ cross-sectional image of the liquid was obtained using Fourier reconstruction. Data was captured as one read frequency encode, and one phase encode, for a resolution of $128 \times 32$, but reconstructed into images of $64 \times 64$. Selected images are shown in figure $2 \mathrm{~b}$, where the sequential melting of the liquid in the $40 \AA, 60 \AA, 140 \AA$ and $200 \AA$ pore diameter silicas can be seen.

The imaging sequence used was modified from being a gradient echo sequence, to one using a $90^{\circ}{ }_{x}-\tau-180^{\circ}{ }^{-}$- -echo sequence, with a $2 \tau$ of $14 \mathrm{~ms}$, as this time was greater than the $\mathrm{T}_{2}{ }^{*}$ of the wide-bore magnet used and was suitably between the decay time in the solid (up to $2 \mathrm{~ms}$ ) and the decay time in the liquid $(500 \mathrm{~ms})$. CYCLOPS phase cycling [8] was used to eliminate D.C. offsets and ghosting due to receiver channel gain differences.

Averaging was kept to the minimum of 4 required by the CYCLOPS sequence, giving a slice imaging time of 4.3 minutes, with a repeat time of 1 second limited by the longitudinal relaxation time $\mathrm{T}_{1}$.

Applying the constraint along the temperature axis that a noiseless signal should only increase in amplitude as the temperature increases and the sample melts, gave a marked improvement in signal to noise (figures $2 \mathrm{~b}, 2 \mathrm{c}$ ). This is particularly important as the next step in the analysis is differentiation along the temperature axis.

The 32 images (averaged in pairs for display purposes) are shown in figure $2 \mathrm{~d}$. The 
cryoporometric differentiation w.r.t. temperature and re-map was then applied to each xy pixel of the data set in figure $2 \mathrm{~d}$. The resulting porosity maps as a function of pore diameter and xy position are shown in figure 2e.

It is possible to pick individual pixels from the data structure of figure $2 \mathrm{e}$, and plot the full pore size distributions for these pixels. This has been done for the four pixels $(22,22)(22,42)(42,22)(42,42)$ (figure 2f). Pore size resolution is comparable with that obtained from standard cryoporometry runs, except at large pore sizes where it can be improved by warming more slowly, thus reducing the thermal gradients that can act to blur the pore size information.

A plot of median pore size for each pixel in figure 2e has also been generated (figure $2 \mathrm{~g}$ ). This provides a concise summary of the average pore sizes present, though containing less information.

\section{3D Resolved Pore Size Structure}

A test phantom was prepared in a $10 \mathrm{~mm}$ diameter tube, that visually appears to be just $15 \mathrm{~mm}$ of silica powder in the bottom of the tube.

The sample was actually prepared using dry $60 \AA$ and $500 \AA$ nominal pore diameter silica as follows : about $5 \mathrm{~mm}$ of $60 \AA$ silica was placed in the bottom of the $10 \mathrm{~mm}$ tube, then $20 \mathrm{~mm}$ of $60 \AA$ silica was placed in a $5 \mathrm{~mm}$ tube, which was then up-ended and pushed to the base of the $10 \mathrm{~mm}$ tube. $500 \AA$ silica was added as a collar, to a further depth of about $5 \mathrm{~mm}$.

The $5 \mathrm{~mm}$ tube was then carefully withdrawn, while tapping, hopefully leaving a central column of $60 \AA$ silica, the excess then spilling over the $500 \AA$ silica to a further depth of about $5 \mathrm{~mm}$. The ideal shape of the $500 \AA$ and $60 \AA$ silica, ignoring mixing of the dry powders, hand shake, etc. is given in figure 3a. The phantom was then fully dried and anhydrous cyclohexane injected into the silica with a hypodermic needle (to facilitate equilibration of the cyclohexane), under dry nitrogen in a glovebox.

The phantom was cooled and 3D image sets captured as a function of temperature. The imaging sequence used was a 3D Fourier transform sequence (one read frequency encode, and two phase encodes) for a resolution of $128 \times 32 \times 32$, but reconstructed into $(64 \times 64) \times 32$ slices at each temperature. Again a spin echo sequence was used with CYCLOPS phase cycling, and averaging was kept to the minimum of 4 required by the CYCLOPS sequence; each 3D image data set took about 60 minutes to acquire, with a repeat time of 1 second.

After extraction of data sets for all the silica, the $500 \AA$ silica and the $60 \AA$ silica, the $3 \mathrm{D}$ data sets were then rendered with surface recognition and shading (figure $3 \mathrm{~b}$ ), using our own routines [9]. The left hand image is of all the silica powder, with no discrimination. The middle image shows just the $500 \AA$ silica, the right hand image is 
of just the $60 \AA$ silica. The linear features mark where silica powder has been displaced by the hypodermic needle.

It is possible to view the 3D data sets from any direction; a view from below the sample shows the collar of $500 \AA$ silica more clearly (figure $3 \mathrm{c}$ ).

This ability to take a porous solid and to generate images of just those regions whose pore sizes fall within a particular range is a novel capability that must have wide applications in physical, chemical and biological studies.

In conclusion, a method for determining the full pore size distribution and median pore size for any point in a 1 or 2-dimensional sample has been presented. Measurement and shaded surface representation of 3-dimensionally resolved pore size structures has also been demonstrated.

This method offers a uniquely non-destructive method of obtaining the full pore size distribution or median pore size at any point inside a bulk sample. 


\section{Acknowledgments}

The authors thank EPSRC, Unilever Research, Oxford Instruments AIG and SMIS Ltd. for research support.

\section{References}

[1] Jackson C L and McKenna G B 1998 J. Chem. Phys. 93 9002-9011.

[2] Strange J H, Rahman M and Smith E G 1993 Phys. Rev. Let. 7121 3589-3591.

[3] Harris R K 1987 Nuclear Magnetic Resonance Spectroscopy, A Physicochemical View. Longman Scientific \& Technical.

[4] Strange J H and Webber J B Characterisation of Porous Solids by NMR, 12th Specialised Colloque Ampere, Corfu 1995.

[5] Norris M O and Strange J H 1969 A Nuclear Magnetic Resonance sample temperature controller using liquid nitrogen injection. J.Phys. E, 2, 2, 1106-1108.

[6] Callaghan P T 1993 Principles of Nuclear Magnetic Resonance Microscopy. Oxford Science Publications, Clarendon Press, Oxford.

[7] Kroon D J 1968 Laboratory Magnets. Philips Technical Library, Eindhoven, 186-189.

[8] Hoult D I and Richards R E 1975 Proc. Roy. Soc. (London) A344 311.

[9] Green T A P 1994 M.Sc. Thesis, University of Kent. 